\title{
Biological Evaluation of Multivalent-Type $N$-Acetyl-D-Glucosamine (GIcNAc) Conjugates for Wheat Germ Agglutinin (WGA) by the Surface Plasmon Resonance (SPR) Method
}

\author{
Amrita Kumari ${ }^{1}$, Tetsuo Koyama ${ }^{1}$, Ken Hatano ${ }^{1}$ and Koji Matsuoka* \\ ${ }^{1}$ Division of Material Science, Graduate School of Science and Engineering, Saitama University, Sakura, Saitama 338-8570, \\ Japan.
}

Received: 08 December, 2016; Accepted: 20 December, 2016; Published: 30 December, 2016

*Corresponding author: Koji Matsuoka, Professor of Graduate School of Science and Engineering, Division of Functional Material Sciences, Saitama University, Shimookubo, Sakura-Ku, Saitama 338-8570, Japan, Tel. No: +81-48-858-3099, Fax: +81 48 858 3099; E-mail: koji@fms. saitama-u.ac.jp

\begin{abstract}
Analysis of the interaction of synthetic avidin-biotin-GlcNAc (ABG) glycocluster complex with a well-known lectin, wheat germ agglutinin (WGA), was performed with a biosensor based on surface plasmon resonance (SPR). In the SPR measurements, WGA was covalently coupled to the gold surface using the amine-coupling method. Artificial glycopolymers of $N$-acetyl-D-glucosamine, polystyrene-based linear-type glycoclusters with a polymeric backbone of acrylamide, were used as controls. Three glycopolymers, including tetrameric $\mathrm{ABG}$ complex, glycopolymer $\mathbf{1}$ with a monomer and acrylamide ratio of 1:10 and glycopolymer 2 with a ratio of 1:4, were used as analytes. The SPR method was used for the analysis of the interactions that covered a high affinity range; namely, the strong binding of $K \sim 6.45 \times 10^{7} \mathrm{M}^{-1}$ for ABG compared with glycopolymers 1 and 2, which show binding of $K_{A} \sim 3.41 \times 10^{5} \mathrm{M}^{-1}$ and $K_{A} \sim 3.30 \times 10^{5} \mathrm{M}$ ${ }^{1}$ respectively. SPR measurements confirmed that WGA has higher affinity toward the tetrameric $\mathrm{ABG}$ complex than toward the lineartype glycopolymers $\mathbf{1}$ and $\mathbf{2}$, and the usefulness of these synthetic glycopolymers as tools in the study of sugar-lectin interactions has been proved due to the very well-known "glycocluster effect".
\end{abstract}

Keywords: Surface plasmon resonance; Glycocluster effect; Polystyrene; Analyte; Amine coupling; Multivalent

\section{Abbreviations}

GlcNAc: $\quad N$-Acetyl-D-glucosamine, GPC: Gel Permeation Chromatography, WGA: Wheat Germ Agglutinin, SPR: Surface Plasmon Resonance, ABG: Avidin-Biotin-GlcNAc, RU: Resonance Units, PL: Photo Luminescence, PBS: Phosphate Buffer Saline, ELLA: Enzyme-Linked Lectin Assay

\section{Introduction}

Carbohydrates present on the cell surface play a vital role in cell recognition, and they help to protect the cell from the outside world and provide biological information. The phenomena of cell adhesion and cell activation prompted by carbohydrate-protein interaction are among the recent topics of active research. Synthetic polyvalent glycoconjugates that imitate the cell surface glycocalyx have been focused on due to their fascinating biological properties [1-2]. In contrast to the weak and poorly specific interactions that arise between individual carbohydrates and proteins, the multivalent display of carbohydrates at the surface of a molecular scaffold is currently used to boost the binding avidity and selectivity toward a target protein $[3,4]$. This phenomenon is known as "cluster glycoside effect" [5-7].

Lectins are inherently proteins of a non-immune source that recognize and bind to specific saccharide structural epitopes present on the surface of a cell membrane [8]. Many of the proteins that participate in multivalent interactions are oligomers such as the lectin wheat germ agglutinin (WGA), an $\mathrm{N}$-acetyl-D-glucosamine (GlcNAc)-specific plant lectin that is extensively used in model studies with glycopolymers to monitor their binding selectivity. WGA is a $36-\mathrm{kDa}$ dimer protein with eight specific binding sites for GlcNAc that are separated by a distance of $14 \AA[9,10]$. GlcNAc is important in several biological systems. It has been proposed as a treatment for autoimmune diseases, and recent tests have claimed some success [11]. The most important thing about GlcNAc is that it is highly specific towards binding with the plant lectin WGA [12]. This is the reason we have chosen WGA as a model lectin and GlcNAc as a sugar model to do further biological research in this project.

The difficulty in studies on carbohydrate-protein interactions is that binding affinities are very weak, usually with dissociation constants in the millimolar range [13]. This limitation is repeatedly overcome by derivatization of carbohydrates to obtain higher binding values due to the phenomenon of multivalency or polyvalency.

Koivula and co-workers confirmed by using SPR technology that WGA has higher affinity toward self-assembled monolayers 
(GlcNAc-SAM) than toward free GlcNAc monosaccharide [14]. Wittman and co-workers developed a library of cycloheptapeptides exhibiting up to six GlcNAc moieties through a urethane spacer. Enzyme-linked lectin assay (ELLA) with WGA has enabled the identification of tetra-, penta-, and hexavalent glycocluster exhibiting higher binding affinity than the monomeric GlcNAc control [15]. On the basis of structural data and recent progress made in the understanding of multivalent effects, a large variety of synthetic glycoclusters based on peptide dendrimers [16], carbosilanes [17], and nanostructures [18] have been developed as ligands for studying biologically relevant targets or providing compounds with anti-pathogenic and anti-tumoral properties.

The availability of radical polymerization approaches, mild reaction conditions and facile purification steps has enabled the synthesis of glycopolymers 1 and 2 in an aqueous solution [19]. Moreover, a tetrameric ABG complex could be prepared by glycosylation steps followed by coupling reaction with biotin and finally avidin-biotin conjugation [20]. Such synthetic multivalent glycopolymers can bind specifically to WGA with enhanced affinity compared to GlcNAc only.

We present here data on the interaction between the plant lectin WGA and synthetic GlcNAc polymers $[19,20]$ by finding the kinetics/affinity using surface plasmon resonance (SPR). SPR is a highly sensitive and powerful technique that has been used in mechanistic studies of carbohydrate-protein interactions at interfaces in real time and in a quantitative manner [21, 22]. SPR biosensing can provide data with desirable reproducibility and therefore offers the possibility of detailed computational analysis.

\section{Results}

\section{Synthesis of glycopolymers}

To obtain multivalent carbohydrate-protein interactions, we synthesized a glycopolymer of a tetrameric structure $A B G$ complex by the use of 4-(4,6-dimethoxy-1,3,5-triazin-2-yl)-4methylmorpholinium chloride (DMT-MM) as a coupling reagent followed by biotin-avidin complexation [Figure 1], and then structurally examined by NMR, IR, MS and elemental analysis [20].

The other two glycopolymers, $\mathbf{1}$ and $\mathbf{2}$, were synthesized by the use of 4-(chloromethyl) styrene, and further azidation followed by reduction reactions were able to yield amino styrene. Condensation of amine with GlcNAc monomer was accomplished and it was polymerized with acrylamide to yield the corresponding water-soluble glycopolymers. These glycopolymers were structurally examined by NMR, IR, MS and gel permeation chromatography (GPC) [19].

\section{Surface Plasmon Resonance Studies}

Despite the fact that SPR has been used for the analysis of carbohydrate-lectin binding, lectin was often immobilized on the sensor surface instead of the glycan moiety [23] because of the low sensitivity inferred by the low molecular weight of the glycan. In order to compare the bindings of different carbohydrate derivatives containing $\mathrm{N}$-acetyl-D-glucosamine to WGA, we immobilized lectin on the sensor chip.

\section{pH scouting}

The surface concentration of the ligand at $\mathrm{pH} 5.5$ was higher (RU 29783.4) than that at $\mathrm{pH} 5.0,4.5$ or 4.0 , and pH 5.5 therefore appeared to be the best choice [Figure 2]. Thus, for immobilization of WGA, pH 5.5 (a value less than pI $8.7 \pm 0.3$ of WGA) [24, 25] was chosen for further modification of the sensor chip.

\section{Immobilization of WGA on a CM5 chip}

The experimental sensor chips (CM5 chips) were modified with a covalently bound ligand (WGA) via amine coupling by $0.4 \quad \mathrm{M} \quad[N$-ethyl- $N$ '-(3-dimethylaminopropyl)-carbodiimide hydrochloride] (EDC) and 0.1 M N-hydroxysuccinimide (NHS) derivatization. After activation, WGA was immobilized by injection of solutions of $36 \mu \mathrm{g} / \mathrm{mL}$ and $720 \mu \mathrm{g} / \mathrm{mL}$ to the respective sensor surfaces, followed by deactivation of residual NHS esters with $1 \mathrm{M}$ ethanolamine. Upon immobilization of

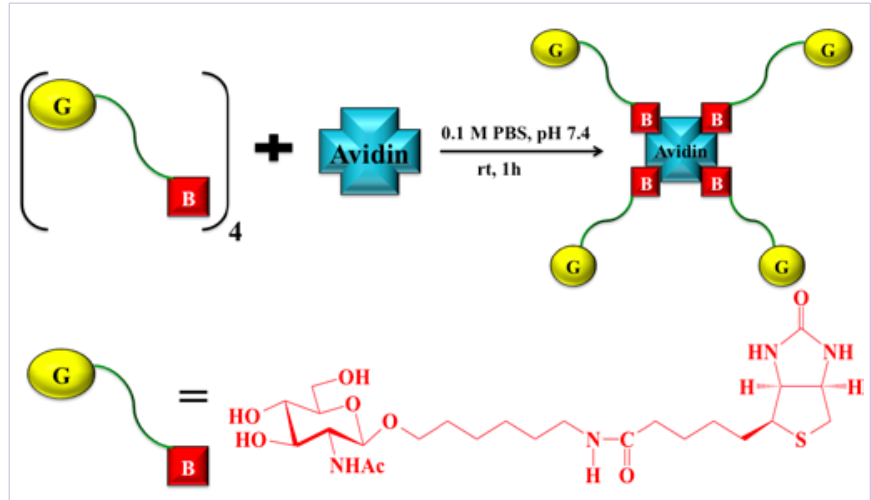

Figure 1: Model representation of the synthetic assembly of avidin-biotin-GlcNAc (ABG) complex [20]. Four units of biotin-GlcNAc conjugate are stirred with avidin for $1 \mathrm{~h}$ at room temperature in the presence of PBS buffer

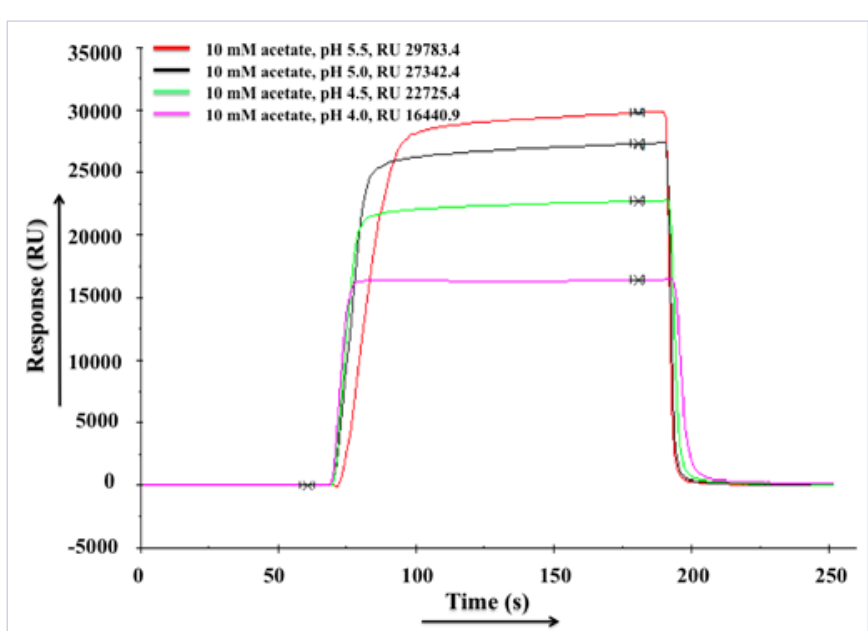

Figure 2: "pH scouting" of WGA Sensorgram showing a relative response-time graph in real time 
approximately $50 \mathrm{RU}$, the final response achieved was $68.50 \mathrm{RU}$ on one chip, and another chip was targeted with $1000 \mathrm{RU}$, the final response achieved being 695.6 RU [Table 1].

Sensorgrams in Figure 3 show the immobilization of WGA on the flow channel of the CM5 sensor chip via amine coupling at $\mathrm{pH}$ 5.5 with $10 \mathrm{mM}$ sodium acetate buffer. The procedure includes the following steps. (A) A "pre-concentration" test is carried out to determine the appropriate ligand concentration to inject in order to reach the targeted level of response (RU, response units). Here, RU are 50 in the case of Figure 3a and 1000 in the case of Figure $3 \mathrm{~b}$ The injection period ends after acquisition and completion of pre-concentration analysis above the baseline of approximately 23,000 [Figure 3a] and 16,500 [Figure 3b]. (B) "Rinse with buffer" involves washing the non-covalently bound ligand with $50 \mathrm{mM} \mathrm{NaOH}$ to completely remove the ligand and obtain the baseline. (C) "Activation" involves injection of the coupling reaction mix (NHS/EDC) onto the surface, activating the carboxymethyl group by forming a highly reactive succinimide ester, followed by termination of the injection. (D)There is a slight increase in RU reflecting the activation, compared to the baseline between B and C. (E) Surface activation is followed by injection of a ligand sample diluted in $10 \mathrm{mM}$ sodium acetate $\mathrm{pH}$ 5.5 buffer, with continuation of injection resulting in covalent binding of the ligand to the reactive surface to yield the targeted RU of 50 [Figure 3a] and 1000 [Figure 3b]. These values are the difference between the achieved constant level just before $F$ and the baseline at D. (F) The remaining non-reacted activated carboxymethyl groups are blocked by injection of ethanolamine, followed by cessation of injection. (G) Immobilization of the ligand is achieved.

\section{Kinetic analysis}

Various sensorgrams can be acquired at different concentrations of the injected compound and they can be simultaneously used to obtain precise kinetic $(k)$ and equilibrium $(K)$ constants. Equilibrium constants can be derived independently from ratios of rate constants or by fitting the

Table1: Kinetic parameters obtained from the interactions of lectin (WGA) with artificial GlcNAc polymers by use of a biosensor SPR technique. The closeness of fit is indicated by the value of $\chi 2$

\begin{tabular}{|c|c|c|c|}
\hline Compound & ABG complex & GlcNAc (1:10) & GlcNAc (1:4) \\
\hline $\begin{array}{c}\text { Molecular weight (Da) } \\
\text { Immobilized ligand } \\
(\mathrm{RU})\end{array}$ & 71186.72 & 1238.37 & 740.81 \\
\hline $\mathrm{K}_{\mathrm{D}}[\mathrm{M}]$ & $1.55 \times 10^{-8}$ & $2.99 \times 10^{-6}$ & $3.05 \times 10^{-6}$ \\
\hline $\mathrm{K}_{\mathrm{A}}\left[\mathrm{M}^{-1}\right]$ & $6.45 \times 10^{7}$ & $3.41 \times 10^{5}$ & $3.30 \times 10^{5}$ \\
\hline $\mathrm{K}_{\mathrm{a}}\left[\mathrm{M}^{-1} \mathrm{~s}^{-1}\right]$ & $1.85 \times 10^{5}$ & $0.70 \times 10^{3}$ & $0.80 \times 10^{3}$ \\
\hline $\mathrm{K}_{\mathrm{d}}\left[\mathrm{s}^{-1}\right]$ & $2.86 \times 10^{-3}$ & $2.05 \times 10^{-3}$ & $2.44 \times 10^{-3}$ \\
\hline${\text { Theoretical } \mathrm{R}_{\max }}^{\text {Calculated } \mathrm{R}_{\max }}$ & 135.4 & 24.0 & 14.3 \\
\hline$\chi^{2}$ & 118.1 & 20.2 & 19.0 \\
\hline & 3.0 & 2.1 & 1.1 \\
\hline
\end{tabular}
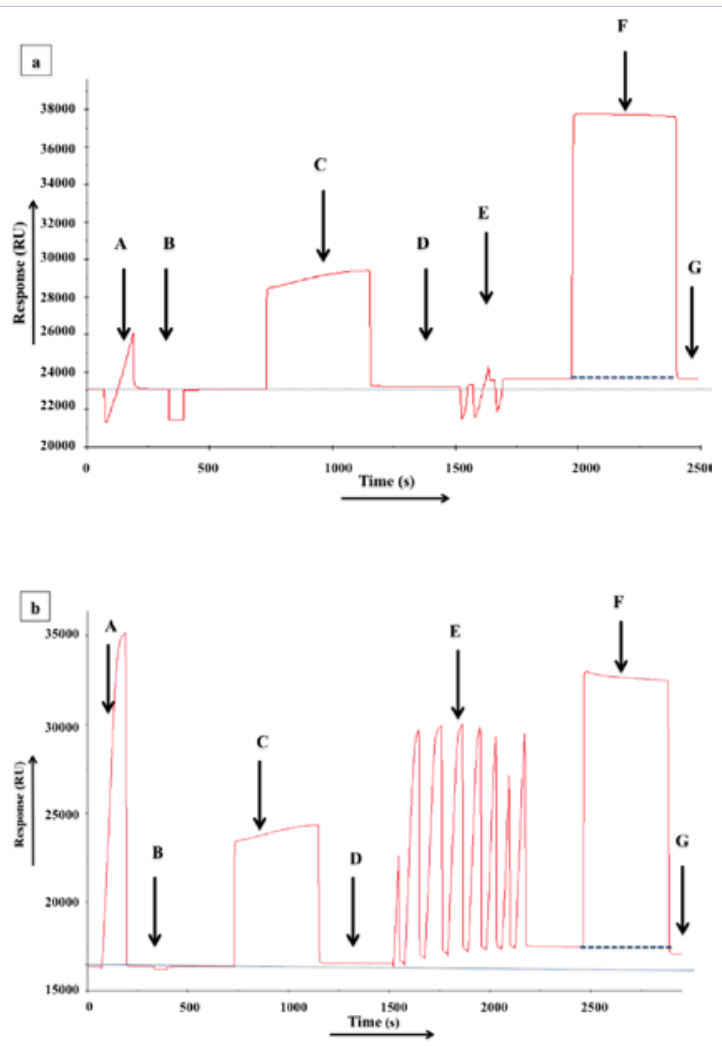

Figure 3: WGA immobilization via amine coupling steps: (A) pre concentration, (B) Washing with $50 \mathrm{mM} \mathrm{NaOH}$, (C) NHS/EDC activation, (D) RU reflecting the activation, (E) amount of ligand bound, (F) Ethanolamine injection and (G) immobilized ligand

steady-state response versus the concentration of the binding molecule in the flow solution over a range of concentrations. SPR signal alteration is a wonderful method to conclude binding stoichiometry, since the refractive index change in SPR experiments produces basically the identical response for each bound molecule and depends on the molecular weight of the binding molecules. Kinetic analysis was performed by injection of analytes of ABG complex and GlcNAc polymers 1 and 2 dissolved in HEPES buffer at different concentrations on a WGAimmobilized chip. Between binding cycles, the WGA surface was regenerated with a 30 s pulse of $200 \mathrm{mM}$ EDTA [26, 27]. Maximum responses of the glycopolymers to the surface-bound WGA were analyzed and plotted against glycoside concentrations [Figure 4]. All of the three glycopolymers showed binding to WGA. Figure 4 a shows that the bound relative response (RU) of $A B G$ complex is high even at low concentrations of ( $4.30-340 \mathrm{nM})$ because of its strong binding to WGA due to its symmetrically arranged tetrameric structure. However, the binding of glycopolymers 1 and 2 was small even at high concentrations $(\sim 100 \mu \mathrm{M})$ compared to $\mathrm{ABG}$. The binding of tetravalent $\mathrm{ABG}$ complex is approximately 190-times higher than that of glycopolymers $\mathbf{1}$ and $\mathbf{2}$, and this difference is explained by the "glycocluster effect".

Some strange inflections can be seen in Figure 4, and they may have been due to the bulk effect $[28,29]$. Such effects basically occur if the running buffer and analyte dilution buffer 
are not the same. The response in Biacore is the extent of refractive index change at the surface of the sensor chip. The variation in refractive index between the dilution buffer and the baseline buffer (running buffer) is called the "bulk effect" and is induced by the existence of dissolved material including buffer components, biomolecules and salt. Samples should be prepared in running buffer to avoid bulk effects during the injections due to differences in the refractive index between the running buffer and sample. The ABG analyte stock was prepared in $10 \mathrm{mM}$ PBS pH 7.4 containing $\sim 137 \mathrm{mM} \mathrm{NaCl}$, while stocks of the other two analytes, glycopolymers $\mathbf{1}$ and $\mathbf{2}$, were prepared in MilliQ and then three-fold dilutions were made in running buffer $(10 \mathrm{mM}$ HEPES, pH 7.4 containing $500 \mathrm{mM} \mathrm{NaCl}$ ), though the dilution buffer and running buffer we used were the same. However, the first higher concentration we used for ABG during the assay contain a mixture of PBS and HEPES buffer and we therefore found some spikes at the end of injection as the buffers contain different salt concentrations.

\section{Surface regeneration}

The affinity between immobilized lectin and glycoclusters used in this study seems to be higher $\left(\sim 10^{7}\right.$ and $\left.10^{5} \mathrm{M}^{-1}\right)$ than the monosaccharide's affinity $\left(\sim 10^{3} \mathrm{M}^{-1}\right)$, and this strong binding was dissociated by a special regeneration solution (10 mM HEPES with 200 mM EDTA, pH 7.4 to chelate bivalent $\mathrm{Ca}^{2+}$ [ $[26,27]$ to obtain an accurate dissociation rate constant. Regeneration at $30 \mu \mathrm{L} /$ min was performed after every cycle of analyte bound to the surface and efficacy of the surface was maintained. The Regeneration process allows the sensor chip to be reused, thus reducing the cost of SPR analysis. However, the lectin surface should be intact without any damage or inactivation of the surface. The regeneration step shortens the analysis procedure as the chip can be used several times without a further immobilization process.

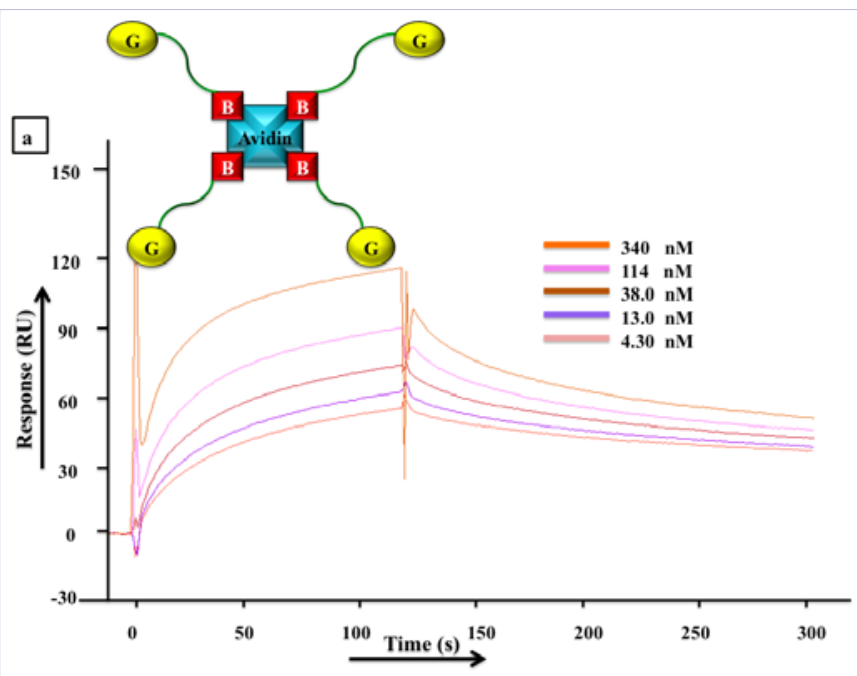

Figure 4: Sensorgrams show interactions of synthetic glycopolymers with lectin WGA. Sensorgrams of the interactions show each association and dissociation phase as a relative response of SPR against time. (a) ABG tetramer complex (4.30-340 nm), (b) glycopolymer 1 (0.153-113 $\mu \mathrm{M})$, (c) glycopolymer $2(0.23-54 \mu \mathrm{M})$

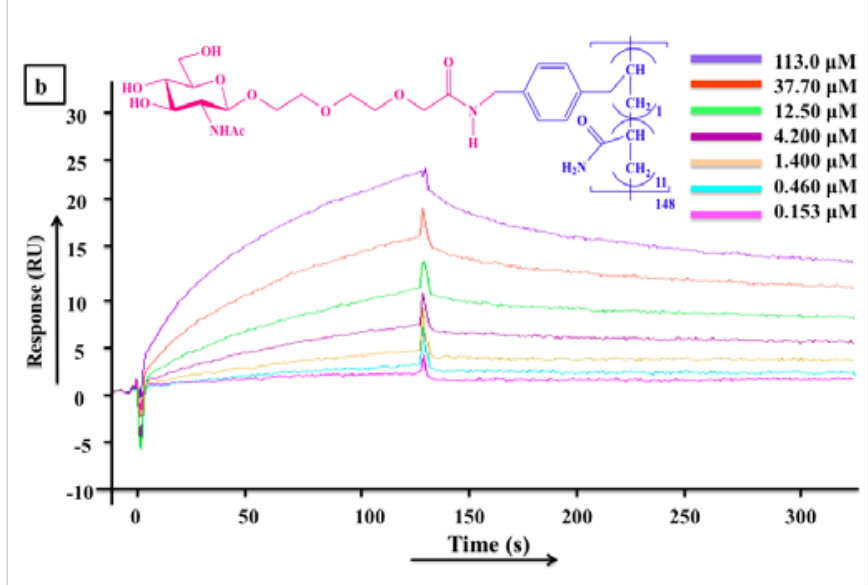

Figure $4 b$

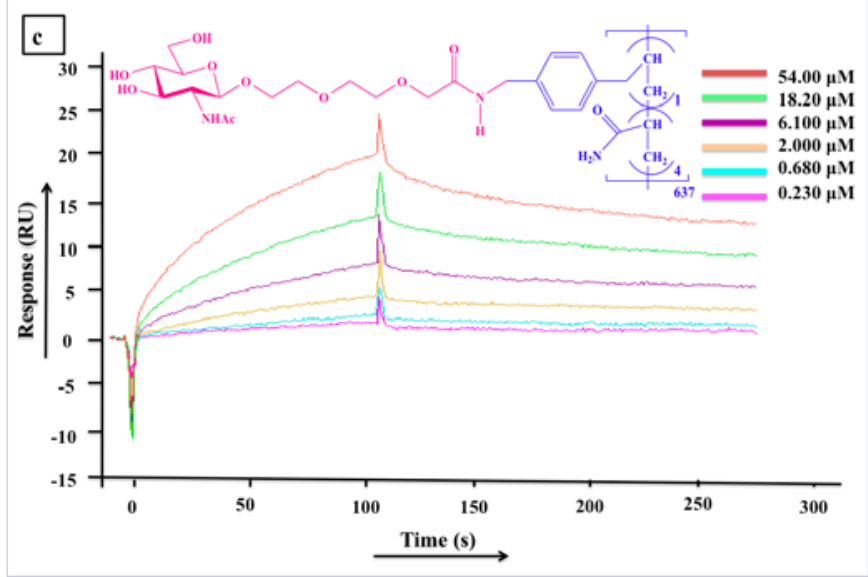

Figure 4c

\section{Kinetic parameters}

We calculated dissociation constants $K_{D^{\prime}}$ which indicate the concentration of the analyte in the equilibrium state. We performed this calculation by fitting each measured value to a single site interaction model, which is a common procedure used in this type of experiment [30]. The results were analyzed by BIAevaluation software and are summarized in Table 1 . As can be seen, different $K_{D}$ and $K_{A}$ values were obtained for different glycol conjugates. Interestingly, higher binding constants were recorded with $K_{A} \sim 10^{7} \mathrm{M}^{-1}$ in the case of ABG complex, its tetrameric known structure afforded the glycocluster effect in comparison with $K_{A}$ $\sim 10^{5} \mathrm{M}^{-1}$ for the other two linear-type polymers used as controls.

\section{Discussion}

The WGA lectin is a dimer of two identical 18-kDa subunits, each consisting of four homologous domains of 43 amino acids. WGA specifically recognizes GlcNAc. Eight independent carbohydrate-binding sites are present per lectin molecule [9, $10]$ and it is therefore interesting to use such a lectin model. During the pre-concentration procedure performed for WGA, the optimum $\mathrm{pH}$ of sodium acetate buffer was 5.5. This buffer 
was therefore used for immobilization of the lectin in the working channel, resulting in an SPR baseline rise by $\sim 700$ $\mathrm{RU}$ (target of $1000 \mathrm{RU}$ )on one sensor chip and $\sim 70 \mathrm{RU}$ (target of $50 \mathrm{RU}$ ) on another sensor chip. In the binding experiments with WGA, the ABG glycopolymer was used as an analyte at a concentration of $340 \mathrm{nM}$, which was diluted three fold, and at such low concentrations, it gave higher binding and higher SPR response (118 RU) than those of the other two glycopolymers. The corresponding glycopolymers, $\mathbf{1}$ and $\mathbf{2}$ showed lower SPR responses, which were comparable to the tetrameric $\mathrm{ABG}$ complex. Although the valency is four, the avidin-biotin scaffold of the ABG complex enhances binding affinity 6380 times than GlcNAc only and $~ 190$ times higher than control glycopolymers $\mathbf{1}$ and $\mathbf{2}$ and it is assumed that this exceptionally strong effect is to be due to the phenomenon of chelate binding approach [31-33]. The distance between binding sites of WGA is 13-14 $\AA$ (distances between anomeric oxygen's of two bound GlcNAc residues] [34, 35] and the shortest distance between binding sites appears to be as small as $13 \AA$ A. For this reason WGA binds strongly to most multivalent analogs that can execute an assured degree of chelation and augment the binding with every valency [36]. The other two glycopolymers ( $\mathbf{1}$ and $\mathbf{2}$ ) are products after radical means of polymerization. These polymers are linear type with pendant top GlcNAc residues and they were used as controls in this study which showed binding affinity towards the WGA $~ 34$ times than GlcNAc only.

\section{Conclusions}

From the results obtained in this study, it can be concluded that the use of the biosensor BIAcore with SPR as a detection method is a powerful method for investigating the interaction between lectins and glycoproteins. We have already demonstrated that tetravalent ABG complex could be easily prepared using avidinbiotin complexation [20]. Experiments with WGA showed that GlcNAc conjugates after organic modifications had higher protein-carbohydrate binding affinity. However, all the synthetic polymers used in this study showed specific and strong $K_{A}$ than their monomeric glycosides, but the ABG complex is more in the favor of glycocluster effect due to its tetrameric structure $\sim K_{\mathrm{A}}=6.45 \times 10^{7} \mathrm{M}^{-1}$. The binding of tetravalent $\mathrm{ABG}$ complex is approximately 190-times higher than that of glycopolymers 1 and 2 and $\sim 6380$-times higher than its monomer, which can be explained by the glycocluster effect. Glycopolymers $\mathbf{1}$ and $\mathbf{2}$ showed $\sim K_{\mathrm{A}}=3.41 \times 10^{5} \mathrm{M}^{-1}$ and $\sim K_{\mathrm{A}}=3.30 \times 10^{5} \mathrm{M}^{-1}$, respectively, as measured by SPR. We compared the kinetic affinity calculated by SPR data with previously determined photoluminescence (PL) or fluorospectrophotometry data for the same sugar conjugates and both methods (PL \& SPR) are almost in the same agreement for protein-carbohydrate kinetics/affinity. The affinity constant for $A B G$ is $1.39 \times 10^{7} \mathrm{M}^{-1}$ [20], while those for the other two glycopolymers were in the range of $\sim 10^{5} \mathrm{M}^{-1}$ as calculated by PL [19]. Little fluctuation in results of affinity constants calculated by two different methods may occur and it should be emphasized that the PL method measures solution-solution interactions, which are different from the solution-solid interactions measured by SPR [37]. The analyte-ligand interactions in our case are uniquely determined, and the kinetic affinity $\left(K_{A}\right)$ for synthetic glycopolymers is concentration-dependent.

TheSPR method has also been shown to be useful for evaluating the interaction of carbohydrates with proteins in a nano-molar range. In summary, carbohydrate-protein interactions are key steps for many physiological and pathological events. Hence, the development of new carbohydrate conjugates and microarrays is important for detecting these activities by using biophysical methods. Such studies are now concerned to the synthesis of more complex and relevant structures and the study of their biological properties.

\section{Methods \\ SPR measurement}

All SPR measurements were carried out on a BIAcore $\mathrm{X}$ 100 instrument (GE Healthcare) by using a CM5 sensor chip at $25{ }^{\circ} \mathrm{C}$ at a flow rate of $30 \mu \mathrm{L} / \mathrm{min}$. HEPES buffer of $\mathrm{pH} 7.4$ used for all measurements consisted of $10 \mathrm{mM} \mathrm{N}$-(2-hydroxyethyl) piperazine- $N$ '-(2-ethanesulfonic acid) (HEPES), $500 \mathrm{mM} \mathrm{NaCl}$, and $0.02 \%$ Tween 20 (p20) detergent. To enhance the lectincarbohydrate binding, $5.0 \mathrm{mM} \mathrm{CaCl}_{2}$ [38] was also added to the running buffer. SPR experiments were carried out with immobilized WGA using different glycoconjugates as analytes to determine the binding constants. The analytes used in this study were divergent-type $A B G$ glycocluster and linear-type glycopolymers $\mathbf{1}$ and 2 . The stock of ABG complex (1.028 $\mu \mathrm{M}$ ) was prepared in phosphate buffer saline (10 mM PBS, pH 7.4), and $150 \mu \mathrm{M}$ stock solution of glycopolymer 1 and $100 \mu \mathrm{M}$ stock solution of glycopolymer 2 were prepared in MilliQ. Then subsequent 3-fold dilutions of these polymers were prepared in $10 \mathrm{mM}$ HBS-P, pH 7.4 buffer in a total amount of $200 \mu \mathrm{L}$. An aliquot of the solutions $(200 \mu \mathrm{L})$ was then injected over the immobilized chip at a flow rate of $30 \mu \mathrm{L} / \mathrm{min}$ with contact time of $120 \mathrm{~s}$ and dissociation time of $180 \mathrm{~s}$. These parameters were the same for all glycopolymers. The chip was regenerated by injection of 30 $\mu \mathrm{L} / \mathrm{min}$ of HBS-EP+ containing $200 \mathrm{mM}$ of EDTA, pH 7.4. The binding assay was performed by a multi-cycle kinetic (MCK) approach. The binding assay also included three startup cycles using running buffer to equilibrate the surface as well as a zero concentration (30\% running buffer plus $70 \%$ milliQ) cycle of the analyte in order to have a blank response usable for reference subtraction. Each sensorgram was obtained by subtracting the reference cell: buffer only injection and glycopolymer injection without ligand immobilization were performed.

\section{Data analysis}

Data analysis was conducted with the software BIAcore X 100 evaluation (version: 2.0.1).

\section{Acknowledgments}

The authors would like to thank Dr. Hidenao Arai of Graduate School of Science \& Engineering of Saitama University for his valuable advice regarding SPR and BIAcore experiments. The authors are grateful to Rotary Yoneyama Memorial Foundation, 
Japan for continuous support and encouragement. This work was partly supported by a grant-in-aid from Saitama Prefecture (K.M.) (Saitama Leading Edge Project).

\section{Author Contributions}

A.K., T.K., K.H. and K.M. conceived and designed the experiments; A.K. performed the experiments and analyzed the data; A.K., T.K., K.H. and K.M. contributed to preparation of the paper; and A.K. mainly wrote the paper. This study is a PhD work of A.K. under the supervision of K.M.

\section{Conflicts of Interest}

The authors declare no conflict of interest.

\section{References}

1. McEver RP, Moore KL, Cummings RD. Leukocyte trafficking mediated by selectin-carbohydrate interactions. J Biol Chem. 1995;270(19):11025-8.

2. Walsh G, Jefferis R. Post translational modifications in the context of therapeutic proteins. Nat Biotechnol. 2006;24(10):1241-52. doi: $10.1038 / \mathrm{nbt} 1252$.

3. Mammen M, Choi SK, Whitesides GM. Polyvalent interactions in biological systems: Implications for design and use of multivalent ligands and inhibitors. Angew. Chem. Int. Ed. Engl. 1998;37(20):27552794.

4. Kiessling LL, Gestwicki JE, Strong LE. Synthetic multivalent ligands as probes of signal transduction. Angew Chem Int Ed Engl. 2006;45(15):2348-68. doi: 10.1002/anie.200502794.

5. Lee YC, Lee RT. Carbohydrate-protein interactions: Basis of glycobiology. Acc. Chem. Res. 1995;28(8):321-327. doi: 10.1021/ ar00056a001.

6. Matsuoka K, Nishimura S-I. Synthetic glycoconjugates. 5. Polymeric sugar ligands available for determining the binding specificity of lectins. Macromolecules. 1995;28(8):2961-2968. doi: 10.1021/ ma00112a049.

7. Lundquist JJ, Toone EJ. The cluster glycoside effect. Chem Rev. 2002;102(2):555-78.

8. Barondes SH. Bifunctional properties of lectins: lectins redefined. Trends Biochem Sci. 1988;13(12):480-2.

9. Loris R, Hamelryck T, Bouckaert J, Wyns L. Legume lectin structure. Biochim Biophys Acta. 1998;1383(1):9-36.

10. Portillo-Téllez Mdel C, Bello M, Salcedo G, Gutiérrez G, Gómez-Vidales V, García-Hernández E. Folding and homodimerization of wheat germ agglutinin. Biophys J. 2011;101(6):1423-31. doi: 10.1016/j. bpj.2011.07.037.

11. Kamel M, Hanafi M, Bassiouni M. Inhibition of elastase enzyme release from human polymorphonuclear leukocytes by $\mathrm{N}$-acetylgalactosamine and $\mathrm{N}$-acetyl-glucosamine. Clin Exp Rheumatol. 1991;9(1):17-21.

12. Wright CS. Crystal structure of a wheat germ agglutinin/ glycophorinsialoglycopeptide receptor complex. JBiolChem.1992;267(20):1434552.

13. Varki A, Cummings RD, Esko JD, Freeze HH, Stanley P, Bertozzi CR, et al. Essentials of glycobiology, $2^{\text {nd }}$ edition. Cold Spring Harbor (NY): Cold Spring Harbor Laboratory Press; 2009.
14. Lienemann M, Paananen A, Boer H, de la Fuente JM, García I, Penadés $S$, et al. Characterization of the wheat germ agglutinin binding to self-assembled monolayers of neoglycoconjugates by AFM and SPR. Glycobiology. 2009;19(6):633-43. doi: 10.1093/glycob/cwp030.

15. Wittmann V, Seeberger S. Spatial screening of cyclic neoglycopeptides: Identification of polyvalent wheat-germ agglutinin ligands. Angew Chem Int Ed Engl. 2004;43(7):900-3. Doi:10.1002/anie.200352055.

16. Reymond JL, Bergmann M, Darbre T. Glycopeptidedendrimers as Pseudomonas aeruginosabiofilm inhibitors. Chem Soc Rev. 2013;42(11):4814-22. doi: 10.1039/c3cs35504g.

17. Hatano K, Matsuoka K, Terunuma D. Carbosilane glycodendrimers. Chem Soc Rev. 2013;42(11):4574-98. doi: 10.1039/c2cs35421g.

18. Chen Y, Star A, Vidal S. Sweet carbon nanostructures: carbohydrate conjugates with carbon nano tubes and grapheme and their applications. Chem Soc Rev. 2013;42(11):4532-42. doi: 10.1039/ c2cs35396b.

19. Hayama R, Koyama T, Matsushita T, Hatano K, Matsuoka K Chloromethylstyrene as a useful starting material for the preparation of glycopolymers and other functional monomers, under preparation.

20. Kumari A, Koyama T, Hatano K, Matsuoka K. Synthetic assembly of novel avidin-biotin-GlcNAc (ABG) complex as an attractive bio-probe and its interaction with wheat germ agglutinin (WGA). Bioorg Chem. 2016;68:219-25. doi: 10.1016/j.bioorg.2016.08.002.

21. Raman R, Raguram S, Venkataraman G, Paulson JC, Sasisekharan R.Glycomics: an integrated systems approach to structure function relationships of glycans. Nat Methods. 2005;2(11):817-24. doi: 10.1038/nmeth807.

22. Ratner DM, Adams EW, Su J, O'Keefe BR, Mrksich M, Seeberger PH. Probing protein- carbohydrate interactions with microarrays of synthetic oligosaccharides. Chembiochem. 2004;5(3):379-82. doi: 10.1002/cbic.200300804.

23. Haseley SR, Talaga P, Kamerling JP, Vliegenthart JF. Characterization of the carbohydrate binding specificity \& kinetic parameters of lectins by using surface plasmon resonance. Anal Biochem. 1999;274(2):20310. doi: 10.1006/abio.1999.4277.

24. Rice RH, Etzler ME. Chemical modification and hybridization of wheat germ agglutinin. Biochemistry. 1975;14(18):4093-4099. doi: 10.1021/bi00689a027.

25. Monsigny M, Sene C, Obrenovitch A, Roche AC, Delmotte F, Boschetti E. Properties of Succinylated Wheat-Germ Agglutinin. Eur J Biochem. 1979;98(1):39-45.

26. http://www.sprpages.nl/sensor-chips-intro-2/biacore/nta

27. Biacore Sensor Surface Handbook BR-1005-71, Edition AB.

28. MJ O'Brien II, SRJ Brueck, VH Perez-Luna, L Tender, GP Lopez. SPR biosensors: simultaneously removing thermal \& bulk-composition effects. Biosensors \& Bioelectronics. 1999;14(2):145-154.

29. Myszka DG. Improving biosensor analysis. J Mol Recognit. 1999;12(5):279-84. doi: 10.1002/(SICI)10991352(199909/10)12:5<279::AID-JMR473>3.0.C0;2-3.

30. D G Myszka, X He, M Dembo, T A Morton, B Goldstein. Extending the range of rate constants available from Biacore: Interpreting mass transport-influenced binding data. Biophys J. 1998;75(2):583-594. doi: 10.1016/S0006-3495(98)77549-6.

31. Lundquist JJ, Debenham SD, Toone EJ. Multivalency effects in proteincarbohydrate interaction: The binding of the Shiga-like Toxin 1 
binding subunit to Multivalent $C$-linked glycopeptides. J Org Chem. 2000;65(24):8245-50.

32. Kitov PI, Sadowska JM, Mulvey G, Armstrong GD, Ling H, Pannu NS, et al. Shiga-like toxins are neutralized by tailored multivalent carbohydrate ligands. Nature. 2000;403(6770):669-72. doi: 10.1038/35001095.

33. Wittmann V, Pieters RJ. Bridging lectin binding sites by multivalent carbohydrates. Chem Soc Rev. 2013;42(10):4492-503. doi: 10.1039/ c3cs60089k.

34. Wright CS, Kellogg GE. Differences in hydropathic properties of ligand binding at four independent sites in wheat germ agglutininoligosaccharide crystal complexes. Protein Sci. 1996;5(8):1466-76. doi: $10.1002 /$ pro.5560050803.

35. Schwefel D, Maierhofer C, Beck JG, Seeberger S, Diederichs K, Möller
HM, et al. Structural basis of multivalent binding to wheat germ agglutinin. J. Am. Chem. Soc. 2010;132(25)8704-8719. doi: 10.1021/ ja101646k

36. Beckmann HS, Möller HM, Wittmann V. High-affinity multivalent wheat germ agglutinin ligands by one-pot click reaction. Beilstein. Beilstein J Org Chem. 2012;8:819-26. doi: 10.3762/bjoc.8.91.

37. Kulkarni AA, Weiss AA, Iyer SS. Glycan-based high affinity ligands for toxins and pathogen receptors. Med Res Rev. 2010;30(2):327-93. doi: 10.1002/med.20196.

38. Farajollahi MM, Cook DB, Self CH. Self, Major improvement of lectinbased assays through choice of cation and optimization of cation concentration. Anal Biochem. 1998;261(1):118-21. Doi:10.1006/ abio.1998.2563. 